18 | 2014

NOVECENTO... E DINTORNI

Da Torino a Parigi: Laura Malvano storica e critica d'arte

\title{
La Mostra del giardino italiano a Palazzo Vecchio (1931)
}

L'Exposition sur le jardin italien à Palazzo Vecchio (1931)

The Exhibition on the "Italian Garden" in Palazzo Vecchio (1931)

Marilù Cantelli

\section{OpenEdition}

\section{Journals}

Edizione digitale

URL: http://journals.openedition.org/cei/1959

DOI: 10.4000/cei.1959

ISSN: 2260-779X

\section{Editore}

UGA Éditions/Université Grenoble Alpes

\section{Edizione cartacea}

Data di pubblicazione: 30 mars 2014

Paginazione: 233-246

ISBN: 978-2-84310-268-4

ISSN: $1770-9571$

\section{Notizia bibliografica digitale}

Marilù Cantelli, «La Mostra del giardino italiano a Palazzo Vecchio (1931)», Cahiers d'études italiennes [Online], 18 | 2014, online dal 30 septembre 2015, consultato il 27 mars 2021. URL: http:// journals.openedition.org/cei/1959; DOI: https://doi.org/10.4000/cei.1959 


\title{
LA MOSTRA DEL GIARDINO ITALIANO A PALAZZO VECCHIO (1931)
}

\author{
Marilù Cantelli
}

La rilettura di una mostra fiorentina degli anni Trenta è un'occasione per valutare, quasi un secolo dopo, i risultati e i giudizi critici su di un evento culturale fortemente orientato dal particolare contesto nazionalista del momento.

La Mostra del giardino italiano fu inaugurata il 24 aprile 193I nel Salone dei Dugento di Palazzo Vecchio. Un ricchissimo repertorio di 4000 opere ${ }^{\mathrm{I}}$ dedicato alla iconografia storica sul tema del giardino a partire dal medievo fino alla fine del Settecento fu riunito in cinquantadue sale del Palazzo. La selezione comprendeva pitture, disegni, stampe, libri, giuochi, fiori finti antichi e moderni in stoffa, mollica di pane, in filigrana d'argento, perline di porcellana e di vetro provenienti da Doccia e da Murano. L'obiettivo di mostrare in questa occasione che l'arte del giardino italiano era rimasta al centro dell'attenzione europea "per quasi duemila anni» fu alla base dell'importante ed eterogenea raccolta di documenti. Nelle prime linee di presentazione del catalogo, Ugo Ojetti — Presidente della Commissione esecutiva - scriveva:

Con questa Mostra del Giardino Italiano Firenze vuole tornare alle grandi Mostre storiche che, nel I9II con la Mostra del Ritratto Italiano, e nel I922 con quella della Pittura Italiana del '6oo e del '7oo, sono state il suo vanto. Anche questa Mostra intende rimettere in onore un'arte singolarmente nostra che dopo aver conquistato il mondo sembrò offuscata da altre mode o nascosta sotto nomi stranieri ${ }^{2}$.

I. 4000 opere secondo M. TAmassia, Dietro le mostre, Livorno, Sillabe, 2006, p. 8; I 500 opere, secondo Virgilio Vercelloni (ivi, p. 92) che è il solo a dare questa cifra, mentre altri autori confermano il numero di 4000 .

2. Comune di Firenze, Mostra del giardino italiano. Catalogo, Firenze, Palazzo Vecchio, I93I, p. 23. 
Le tre mostre furono preparate dall'équipe formata da Ugo Ojetti, Nello Tarchiani e Luigi Dami. Scomparso nel 1925, Dami venne sostituito dal Conte Carlo Gamba Griselli nella mostra del 1931. Si riconosce la loro impronta in ciascuna delle tre mostre dove l'équipe arriva a riunire un numero impressionante di opere nei due palazzi principali di Firenze: 800 quadri a Palazzo Vecchio per la mostra Il ritratto italiano dal Caravaggio al Tiepolo, r 056 quadri a Palazzo Pitti per La pittura italiana del '6oo e '700. Altra caratteristica comune alle mostre, la loro sommaria presentazione in minuscoli cataloghi. Prima si faceva la mostra, poi, eventualmente, se ne faceva un libro qualche anno dopo ${ }^{3}$. Nei primi due casi, alla descrizione minuziosa delle sale del Palazzo in cui le opere venivano esposte faceva seguito, nel catalogo, la lista degli artisti ed i titoli dei quadri senza alcun cenno sugli obbiettivi della mostra. Per presentare le opere riunite nella Mostra della pittura italiana del Sei e Settecento si disposero i quadri nelle sale secondo l'ordine alfabetico degli autori, per ogni pittore vennero indicate le date di nascita e di morte fatte seguire dai titoli delle opere presentate nella sala senza indicare le misure dei quadri, senza nessun cenno biografico o critico sull'artista e la sua opera. L'avvenimento culturale di questa mostra fu la presentazione di diciotto tele di Caravaggio a cui sarà dato grande spazio nel libro sulla mostra, pubblicato due anni dopo.

È a partire dalla terza mostra su Il giardino italiano che Ojetti, che ne è il Presidente, apre il catalogo con tre pagine d'introduzione dove cerca di sviluppare il tema del "giardino italiano», protagonista della mostra. L'omonimo libro pubblicato dall'amico e collaboratore Luigi Dami nel I925 è scelto come filo conduttore. Come per le Mostre del I9II e del I922, Ojetti sottolinea il proposito di imprimere all'esposizione un carattere di Mostra storica, basata su creazioni destinate a rimanere memorabili ed appartenenti ad una realtà italiana effettivamente documentata. Dato l'interesse manifestato fin dagli inizi dal regime per un tipo di cultura legata alla storia, era considerato particolarmente opportuno, in quegli anni di consolidamento del fascismo, porre l'accento sull'impronta della storia. Dare valore alla cultura italiana del passato sotto tutti i sui aspetti compreso quello del giardino o dell'arte dei giardini, era seguire il processo della politica culturale di autocelebrazione adottato in quegli anni dal regime. La dimensione e la sistemazione della mostra vennero affidate ad una équipe composta da storici dell'arte, eruditi locali, notabili, collezio-

3. Due anni dopo la Mostra del I922, viene pubblicato il libro a cura di U. OJetti, L. Dami e N. Tarchiani, La Pittura italiana del Seicento e del Settecento alla mostra di Palazzo Pitti, Milano-Roma, Bestetti e Tumminelli, I924. 
nisti ed artisti, molti dei quali rivestivano incarichi ufficiali nelle città. Tra i collezionisti milanesi troviamo Achille Bertarelli, collezionista di stampe e libri rari, che presta alla mostra molti pezzi della sua collezione, tra i fiorentini il Conte Carlo Gamba Ghiselli, amatore d'arte, amico di Berenson, presidente della fondazione Horne e, come Ojetti e Tarchiani, poligrafo. Nei comitati regionali, insieme ai direttori dei musei di tutte le regioni d'Italia, figuravano pittori, scenografi, architetti come Giò Ponti,Tommaso Buzzi, Ferdinando Reggiori per la Lombardia, Giovanni Chevalley per il Piemonte, Giuseppe Crosa di Vergani per la Liguria, Luigi Piccinato per il Lazio, Enrico Lusini per la Toscana. I rappresentanti dei Comitati Regionali furono invitati da Ojetti a eseguire un censimento dei giardini storici della loro regione.

Per il tipo del Giardino dei romani fino al Giardino fiorentino del Quattrocento si precisano gli oggetti da cercare: arazzi, affreschi, tavole o, in mancanza di altri documenti, anche foto raffiguranti giardini storici. A partire dal Giardino fiorentino del 'soo si richiedono per ogni regione, dipinti, disegni, stampe, giuochi e svaghi della villeggiatura. Saranno esposte nella Sala del Ballatoio giostre appartenenti a raccolte private: una giostra da giardino con due cavalli e due sedioli del '700, un modello di carosello ad argano del Palazzo Reale di Caserta, giochi vari... Non si invitano nei Comitati né giardinieri né agronomi, ma dato che, come scrive Ojetti, «un giardino senza fiori è un volto senza occhi», Ojetti invita Giovanni Negri, insegnante di botanica nell'Università di Firenze. Nella sezione del fiore finto figurano alcune signore, tra cui Donna Fernada Ojetti e la Signora Prampolini. Anche il Conte Gamba si preoccupa di confermare, nel catalogo, l'indirizzo voluto per la mostra. La mostra doveva essere «specialmente storica e didattica», scrive, perchè «si è fatta con quadri, stampe, piante, modelli e fotografie di giardini esistenti o progettati». Di giardini storici infatti non restavano molte tracce ed anche la conoscenza de Il giardino italiano o, piuttosto, del giardino all'italiana come forma artistica, poteva appoggiarsi su poche ricerche apparse in Italia.

La prima publicazione, in Italia, su Il giardino italiano si deve nel I9I5 a Maria Pasolini Ponti che, in un piccolo libro di trenta pagine, segnala la carenza di studi sul tema nel nostro paese e ricorda i principali lavori inglesi e tedeschi: Italian Villas and their Gardens (1904) di Edith Warton, e Geschichte der Gartenkunst (I9I4) di Marie Louise Gothein. L'autrice presenta i caratteri speciali del "giardino italiano da studiare in relazione alla casa ed all'ambiente». Questo principio non lo limita alle grandi dimore ma lo estende agli spazi pubblici e privati delle città. Pasolini Ponti è la prima in Italia a dare una valutazione negativa del giardino paesaggista 
al quale dedica ironiche pagine di critica nel suo libro4. Questa posizione di condanna viene ripresa da Ojetti nella sua introduzione alla mostra del I93I, dove la critica legata a delle ragionevoli considerazioni di pratica progettuale dell'autrice è sostituita da motivazioni ideologiche per stigmatizzare la fine di un savoir faire italiano contaminato da mode straniere. Nella Mostra le idee generali di un giardino italiano, per tradizione simmetrico ed architettonico, saranno presentate nei giardini dei palazzi e delle grandi dimore accuratamente selezionate per l'occasione. Nessun accenno sarà fatto ai giardini urbani pubblici e privati che l'autrice aveva considerato e il cui studio cominciava ad impegnare le forze più creative tra gli architetti interessati alla organizzazione dei parchi e dei giardini nelle città.

Nel 1924 esce Il giardino italiano di Luigi Dami`s. Fornito di un materiale iconografico di 35 I tavole scelte in gran parte tra opere di pittura, il libro propone un'ambiziosa ricostituzione del giardino italiano a partire dal Medioevo sino alla fine del Settecento, secolo che, anche per questo autore, segna l'inizio della decadenza del giardino italiano.Tra le conclusioni scrive:

Alla fine del XVIII secolo il giardino italiano chiude il ciclo delle sue incarnazioni. D'allora in poi i nuovi giardini per un cinquantennio furono costruiti secondo l'ultimo stile: e moltissimi dei vecchi guastati e rifatti. Dopo di che siamo andati miseramente a finire, in Italia e dappertutto, in un eclettismo senza stile ${ }^{6}$.

4. Maria Pasolini Ponti criticava con ironia certe realizzazioni del giardino paesaggista: «Si addossarono gli alberi alla casa in modo da toglierle il sole; i viali svolti secondo il paesaggio immaginato erano ombreggiati a caso, secondo i gruppi di alberi che il quadro pittoresco suggeriva. Si distesero lunghe file di serre, insediandole nelle parti soleggiate, spesso sciupando colle loro linee monotone l'effetto del paesaggio stesso che si era imaginato e voluto. Alle fontane con getti d'acque spruzzanti, circondate da statue, si preferirono dei piccoli laghi, sui quali si specchiasse il salcio piangente. Alle reminiscenze dell'antico Olimpo, che dilettavano gli abitatori del giardino antico, si contrapposero altri ricordi e false rovine; delle grotte appartate racchiudevano un eremita, il quale si alzava minaccioso o benedicente dinanzi a chi disturbava il suo raccoglimento. La natura era gustata soltanto se avvolta in un velo sentimentale. Il lume di luna doveva apparire più bello, se contemplato in un giardino a fianco di una chiesa gotica o sulle sponde di un lago, a ricordo di quelli cantati dai poeti della Scozia. [...] Era questa una natura schietta, veramente libera e sincera?» (M. PAsolini Ponti, Il giardino italiano, Roma, Ermanno Loescher, I915, p. 22).

5. Dopo una formazione letteraria Dami (I882-1926) s'interessa all'architettura. Nominato dal I92I ispettore della sovrintendenza alle antichità e Belle Arti di Firenze, Dami è un fedele collaboratore e amico di Ugo Ojetti che pubblica i suoi articoli su Dedalo di cui Dami è segretario di redazione. Dami pubblica Il giardino italiano a Milano nel 1924 e nel 1925 prende la libera docenza in storia dell'arte. Nella bibliografia del libro di Dami appare l'espressione "giardino italiano» nelle pubblicazioni di: E. Wharton, Italian Villas and their Gardens, London, John Lane, 1904; I. Triggs, The Art of Garden Design in Italy, London, Longmans Green, 1906; G. S. Elgood, Italian Gardens, London, Longmans Green, I907; A. Le Blond, The old Gardens of Italy, London, John Lane, I9I2; J. M. CARTwright, Italian Gardens of the Renaissance, London, Smith, Elder \& C., i9i4; M. Pasolini Ponti, Il giardino italiano, Roma, Ermanno Loescher, i9i5; E. March Phillips, Gardens of Italy, London, 1905; G. Gromort, Jardins d'Italie, Parigi, A. Vincent, 1923.

6. L. Dami, Il giardino italiano, Milano, Bestetti e Tumminelli, 1924, p. 29. 
Le prime linee del libro di Dami aprono con una definizione del termine «italiano» divergente da quella ideologica adottata nella Mostra:

La parola «italiano» ha in questo libro significato stilistico, non geografico. Si vuole infatti qui definire con qualche precisione, nelle loro varie apparizioni e casi varii, quelle forme che, determinate tra il Quattrocento ed il Cinquecento, tra Firenze e Roma, costituirono allora e poi l'arte italiana del giardino ${ }^{7}$.

Immaginata venti anni prima ed in seguito un po' dimenticata, la mostra del I93I richiese pochissimi mesi di preparazione. Ojetti, che in quegli anni svolge una frenetica attività nella letteratura, nella critica d'arte $e$ nell'organizzazione di mostre, mantiene il controllo dell'andamento dei lavori scegliendo come collaboratori principali le persone che abitualmente lavorano con lui, sia nelle riviste che negli affari più personali, come i lavori di sitemazione della sua villa col giardino all'italiana del Salviatino, realizzati da Enrico Lusini. In quegli anni Lusini è l'architetto fiorentino a cui son affidati importanti lavori di restauro dei monumenti della città. Era intervenuto nel restauro del Palazzo dell'Arte della Lana (1905), nei lavori di ripristino del Palazzo Medici e del suo giardino (I9II-I92I), alle scuderie Reali, che dovevano ospitare il nuovo Istituto d'arte a Porta Romana. L'ultimo suo intervento era stato nelle sale del Mezzanino di Palazzo Vecchio.

La mostra del giardino italiano si svolge a Firenze, nel palazzo trasformato in reggia dai Medici e destinato a sede del governo del Regno d'Italia negli anni di Firenze capitale dal 1865 al I870. Gli organizzatori riserveranno alle due città ex-capitali Torino e Firenze gli spazi più importanti al primo piano del Palazzo della Signoria, «una Reggia medicea annidata nell'antico Palazzo del popolo». Si rende omaggio ai Savoia ed alla monarchia riservando alle ville ed ai castelli sabaudi il Salone dei Dugento al primo piano. L'architetto milanese Tommaso Buzzi ${ }^{8}$ disegnerà per questa sala una Mappa con i palazzi e le sontuose ville sabaude, circondate da boschi e ampii giardini. Buzzi, che diventerà in pochi anni l'architetto ufficiale della nobiltà e della borghesia milanese, miniaturizza nella sua Mappa (fig. I) le splendide incisioni del Theatrum Sabaudiae. Già fin dal Sei e Settecento questa serie omogenea di rappresentazioni della «corona di delizie» attorno a Torino evidenzia il suo carattere di propaganda collegato all'affermazione del processo politico di formazione dello Stato

7. Ivi, p. 7.

8. Tommaso Buzzi (1900-I98I) architetto, lavora col gruppo Novecento e con Giò Ponti. Collabora con Ponti sulle pagine di «Domus» e con Ojetti sulle pagine di «Dedalo». Diventerà l'architetto ufficiale della nobiltà e dell'alta borghesia. 
sabaudo. L'architetto Chevalley presentando il programma del Salone sottolinea come queste creazioni destinate ai Duchi e ai Re sabaudi siano state: «affermazione di potenza e di ricchezza, riposi graditi dove potevano colle fastose loro corti, darsi al prediletto esercizio della caccia, ristoro alle gravi cure del governo»?

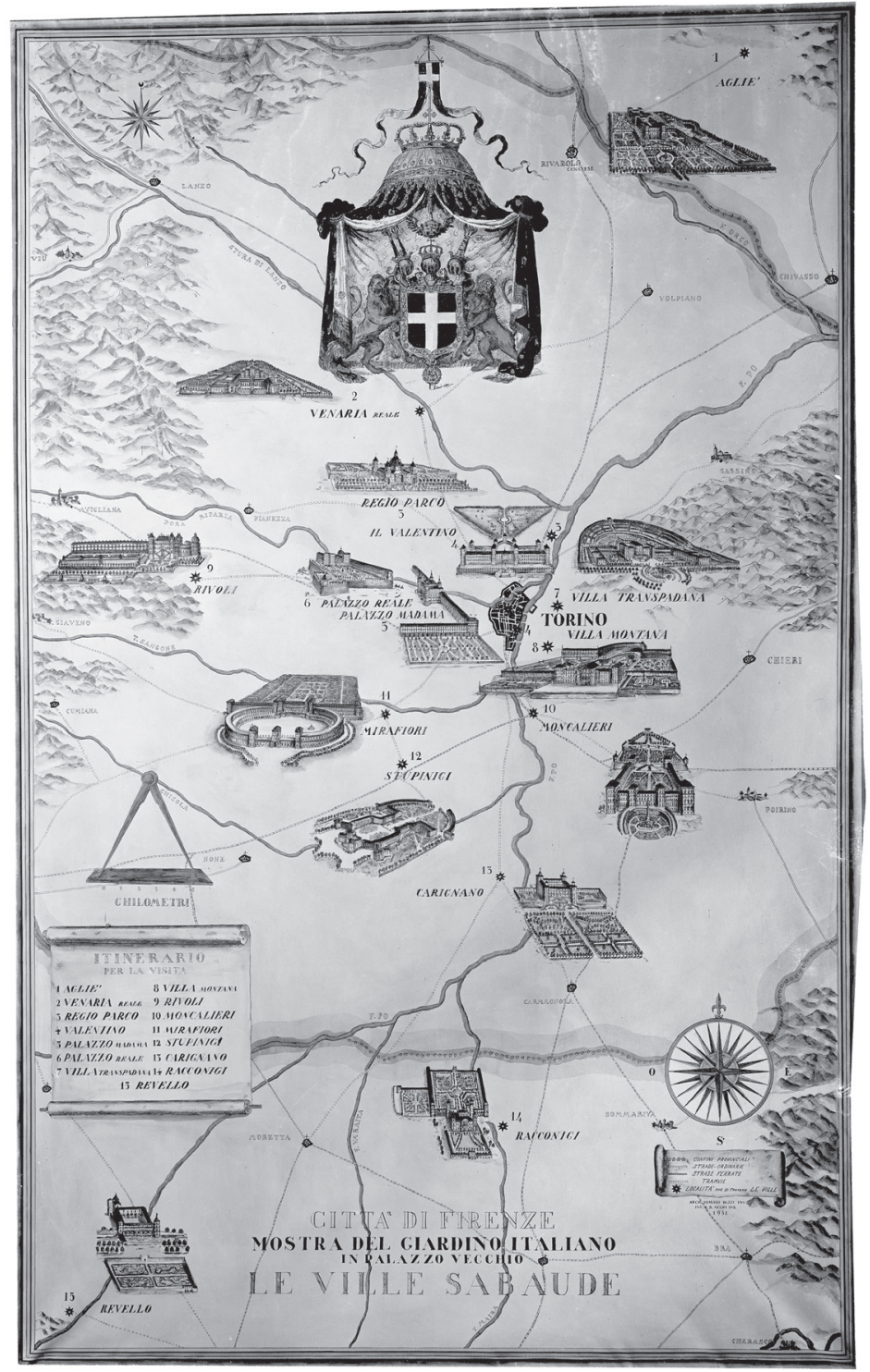

Fig. I. - Le ville sabaude alla Mostra del giardino italiano in Palazzo Vecchio (1931).

9. Mostra del giardino italiano, cit., p. 34 . 
Valorizzare il patrimonio e la committenza delle grandi e nobili famiglie italiane era tra le ambizioni di una buona parte degli organizzatori della mostra. Come ricorda il polemico Persico, lo stesso Ojetti si firmava in certi pezzi "Conte Ottavio» ${ }^{\text {Io }}$. La scelta delle pitture da esporre nel Salone va soprattutto ai ritratti dei nobili proprietari delle ville, dei palazzi, dei castelli i cui giardini sullo sfondo sono visti quasi sempre come spazi di accompagnamento di luoghi appartenenti a personaggi altolocati.

La Toscana ed il Lazio erano stati riconosciuti dal Dami come i luoghi di creazione del giardino italiano prima del suo svilupparsi in tutta Italia. Le ville ed i giardini romani e fiorentini del Cinquecento sono esposti nelle sale del Mezzanino appena restaurate dall'architetto Lusini.

Le Ville Medicee occupano tutte le sale del quartiere di Cosimo. È di nuovo a Tommaso Buzzi cui viene affidato il disegno della Mappa delle Ville Medicee. La cultura letteraria aveva stabilito che il giardino italiano nasce nella Firenze del Rinascimento, nei palazzi di città e nelle ville delle nobili famiglie, soprattutto quella dei Medici.

Dipinti, stampe, disegni, documenti delle Ville Medicee provenienti in gran parte dal Museo Topografico e dal Gabinetto degli Uffizi sono esposti in queste sale del primo piano. Nella piccola Sala degli Otto viene affidata a Giuseppe Lugli la presentazione de Il giardino dei romani. Specialista già consacrato dell'archeologia romana e sempre attento a dare una base scientifica alla ricerca archeologica, Lugli scrive una corta presentazione dove ricorre a fonti letterarie e iconografiche. Nel caso della villa di Plinio scrive prudentemente:

Nei grandi giardini, come ad esempio nella villa di Plinio in Toscana, le piante sapientemente tagliate assumevano le fogge più strane, come figure geometriche (prismi, sfere, coni) oppure animali fantastici, edifici immaginari, navi, paesaggi e perfino episodi interi della guerra troiana e delle peregrinazioni di Ulisse. Questo genere di

Io. Fernando Tempesti ricorda Ojetti come «la bestia nera di Persico», che lo considera «il più autorevole e nefasto critico d'arte del regime fascista». Un attacco di Persico, «al riparo del nome di Bardi», dà un giudizio feroce di Ojetti: «È stato, per principio, amico di tutti, pronto a disfarsi di tutti appena se ne fosse mostrata l'opportunità; con cinquanta vestiti nel guardaroba e un centinaio di cravatte è riuscito a penetrare dovunque, dovunque facendo la figura di persona di casa. Con una giacchetta nera e una cravatta a La Vallière s'è fatto passare per rivoluzionario, con l'abito grigio perla e una cravatta ametista l'hanno scambiato per un elegante stilista dannunziano, con un pastrano all'inglese s'è dato le arie di viaggiatore letteratissimo e aggiornatissimo; fino ad oggi che, con la caramella, dà ad intendere di saperla, ancora, assai lunga. È un piccolo borghese che sentì la necessità di attribuirsi un po' di lustro con la contea dello pseudonimo (Conte Ottavio, firmava Ojetti certi pezzi) [...]. È uno scrittore di giornali borghesi e di riviste con copertine rosa, che ha tentato di mettere il piede anche nella letteratura, nella critica d'arte, nell'organizzazione delle mostre. Ha dannunzieggiato annusando per le peste dell'Imaginifico, e quando Panzini venne di moda panzineggiò; ora sta ritirandosi in pensione con "le cose viste". Ma va detto che ha sempre parlato delle cose a cui non era chiamato e che le sue critiche d'arte hanno lo stile e il decoro delle suonatine ad orecchio. Si spiega così il largo credito borghese che lo deliziò" (F. Tempesti, Arte dell'Italia fascista, Milano, Feltrinelli, 1976, pp. I63-165). 
giardinaggio si chiamava opus topiarum ed era eseguito da veri artisti, ma purtroppo gli elementi che abbiamo non sono sufficienti per tentarne una ricostruzione ${ }^{\mathrm{II}}$.

I documenti che appaiono in questa saletta presentano foto e copie delle decorazioni di giardini e case di Pompei, acqueforti di Piranesi e studi e rilievi degli architetti dell'Accademia Americana a Roma. Ojetti è fiero di questa presenza, testimonianza supplementare dell'interesse portato alla cultura italiana del giardino anche all'estero: «Oggi fuori d'Italia, specie nel Nordamerica - scrive - anche il giardino italiano torna di moda. [...] I più accurati disegni e rilievi moderni delle nostre ville, esposti nella Mostra, sono di architetti americani ${ }^{\mathrm{I}}$.

Il Salone dei Cinquecento viene riservato a quella che doveva essere la parte più spettacolare ed ambiziosa della mostra, la costruzione di dieci tipi di Giardino messi in scena sotto forma di diorama all'interno di altrettanti box di 4,70 x 3 metri, più poeticamente chiamati nel catalogo teatrini. "Questi teatrini con luci acconcie e acque correnti sono stati fino dal primo giorno la grande ammirazione del pubblico» commenta il Conte Gamba un mese dopo l'inaugurazione. La serie di modelli doveva aprirsi col Giardino dei romani per proseguire, seguendo una logica cronologica e geografica, fino al Giardino romantico perchè, come precisa Ojetti, «tutta la evoluzione dell'architettura giardiniera sia, in questa Mostra storica, sotto gli occhi del visitatori ${ }^{13}$.

Lo scenografo fiorentino Donatello Bianchini ${ }^{14}$ e l'architetto Enrico Lusini ${ }^{15}$ si divideranno l'impresa di costruire i dieci giardini ${ }^{16}$ che costituiranno la parte più visitata ed apprezzata dal pubblico, anche se questo programma di giardini mai esistiti contraddiceva la volontà, a più riprese annunciata, di dare alla mostra un carattere «storico». Enrico Lusini fa

II. Mostra del giardino italiano, cit., p. 60.

I2. Ivi, p. 24 .

I3. Ivi, p. 23-24.

I4. Donatello Bianchini, scenografo, collabora alla rivista di Ojetti. Realizza i dieci teatrini della Mostra.

15. Enrico Lusini, architetto e professore dell'Istituto d'arte a Firenze. Gli fu affidata la sistemazione attuale del giardino di Palazzo Medici, nell'ambito del ripristino dell'intero Palazzo (I9II-I92I). L'incarico del giardino di Palazzo Medici gli viene affidato nel I9II dal Presidente del Consiglio Provinciale di Firenze Arturo Linaker che gli fornì importanti informazioni sulle fonti iconografiche e letterarie per il ripristino del giardino. Lusini ne farà tesoro per il suo intervento nella Mostra del I93I dove le fonti che dichiara aver utilizzato sono essenzialmente letterarie.

16. Il giardino dei romani; Il giardino toscano del Trecento; Il giardino fiorentino del Quattrocento; Il giardino fiorentino del Cinquecento, invenzioni dell'architetto Enrico Lusini; Il giardino genovese tra il Cinque e il Seicento, invenzione dell'architetto Giuseppe Crosa di Vergani; Il giardino romano tra Cinque e Seicento, invenzione dell'architetto Luigi Piccinato; Il giardino veneziano del Settecento, invenzione dell'architetto Tommaso Buzzi; Il giardino piemontese del Settecento, invenzione dell'architetto Giovanni Chevalley; Il giardino neoclassico lombardo, invenzione dell'architetto Tommaso Buzzi; Il giardino romantico, invenzione dell'architetto Donatello Bianchini. 
costruire in questa sala una inquadratura per nobilitare ogni contenitore dei dieci diorami, per i quali il catalogo precisa trattarsi di «modelli di Giardini italiani che non riproducono nessun giardino definito, ma vogliono dare al pubblico la rappresentazione essenziale dei tipi più caratteristici, nelle varie epoche, del nostro Giardino ${ }^{17}$.

Lusini è il principale responsabile delle realizzazioni del Salone dei Cinquecento, e l'«inventore» dei primi quattro dei dieci giardini. Per Il giardino dei Romani dichiara d'ispirarsi ai "giardini pompeiani più noti e ammirati ed agli affreschi antichi che in Roma raffigurano giardini», mostrando di dimenticare le prudenti considerazioni di Giuseppe Lugli sull'affidabilità dei riferimenti iconografici. Per Il giardino Toscano del Trecento Lusini dice interpretare il giardino che Boccaccio descrive nel proemio della Terza giornata del Decamerone, secondo un topos adottato da molti di coloro che allora ed anche ai nostri giorni, vogliono rievocare il giardino del Medioevo. La restituzione di Lusini de Il giardino fiorentino del Quattrocento (fig. 2) si appoggia su una ricerca di varie fonti, stimolate dal libro di Dami che, nel capitolo sul «Il Quattrocento», aveva scritto: «Non abbiamo documentazioni precise dei giardini di Cosimo il Vecchio, salvo per quello assai piccolo di città, al palazzo di via Larga».

Durante i lavori di restauro al giardino del Palazzo Medici, Lusini era venuto a conoscenza di fonti rare come la descrizione quattrocentesca dell'Avogadro del giardino di Cosimo nel palazzo di via Larga, grazie alle ricerche del Presidente del Consiglio provinciale di Firenze Arturo Linaker ${ }^{18}$ su I restauri a Palazzo Medici che lo studioso aveva pubblicato. Nella relazione sui lavori al Palazzo, Linaker aveva inserito due fantasiosi poemetti, uno di un poeta contemporaneo di Michelozzo, l'altro del contemporaneo di Cosimo il Vecchio, Albertus Avogadrius Vercellensis che celebrava in versi il giardino di via Larga come un giardino meraviglioso:

Multa mihi scripta sunt; tandem veniamus ad hortum

cui Latio haud nullum non reor esse parem.

Hortus enim est magnus, sed nec pro fructibus actus

Sed quo sit tantum buxea planta decens.

Quam verti in varias cogit manus apta figuras

Hoc elephans, hinc, est dente timendus aper.

Videris et puppim velis maris aequora passis

17. Mostra del giardino, cit., p. 28.

18. A. Linaker, I restauri di Palazzo Medici Riccardi, Firenze, Tip. L'arte della stampa, I9I5. Il poeta contemporaneo di Michelozzo diceva dell'orto: «E ha questo palazzo un bel giardino / Chon corte, logge, volta e acqua e prato / E fu fatto e fiorito in un mattino / Ed è di sì gientile ordini ornato / Di lauri, mortella, aranci e bossi / Ch'un mostro mostra ciò che v'è piantato». 
Scindere, nes cedunt prora rudensque rati.

Illic est aries, illic lepus auribus altis,

Illic est vulpes fallere docta canes;

Cornibus arboreis fas est te visere cervos

Et quae fert centum lumina cernis avem.

Quid moror? hic tot sunt brutorum corpora buxo,

Et diversa quidem, quot reperire licet.

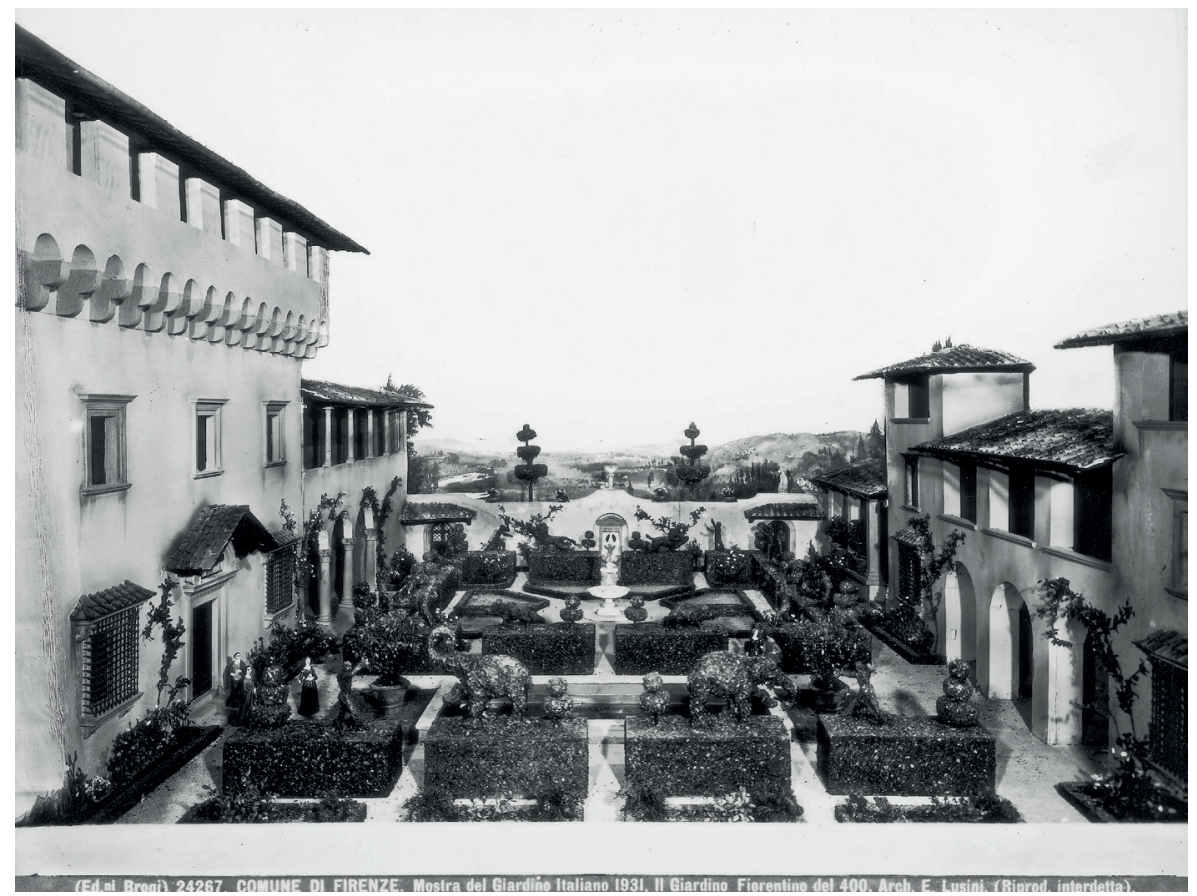

Fig. 2. - Il giardino fiorentino del Quattrocento di E. Lusini.

Visibilmente affascinato dal racconto imaginifico del giardino di Cosimo, Lusini testimonia questa volta il suo desiderio di interpretare il mistero e la magia proprie alla cultura fiorentina e medicea dell'umanesimo con una realizzazione capace di suscitare stupore e meraviglia. La sua versione idealizzata del giardino del Quattrocento offre al pubblico della Mostra un giardino geometrico, ovvero un orto non idoneo a produrre frutti ma ornato di figure dove elefanti, un cinghiale, un ariete, una lepre con le orecchie dritte, volpi, cervi, forme geometriche di varie dimensioni scolpite in finto bosso, reinventano le figure fantasiose del poemetto dell'Avogadro. Il modello del giardino è inserito, come la mostra richiedeva, all'interno di prospetti architettonici ispirati alle ville di Michelozzo. 
Una reinvenzione del leggendario giardino suscitata da favole e miti che attestano la loro capacità di mantenere vivo l'interesse per opere rimaste a lungo nel mistero e nell'oblio ${ }^{19}$.

Per le «invenzioni» degli altri giardini gli architetti incaricati traggono ispirazione piuttosto da realizzazioni o da personalità artistiche che nel passato avevano lasciato la loro impronta nella regione a loro affidata. Il giardino genovese tra il Cinque e Seicento di Crosa Vergani - architetto di ville e giardini destinati alla classe dirigente genovese - è un richiamo alle ville dell'Alessi: «la Villa Imperiale a Sampierdarena», la Villa Cambiaso e la Villa Franzone; Piccinato che in quegli anni pur s'interessa ad un nuovo progetto d'inserimento e di ridistribuzione dei parchi e giardini nel tessuto urbano di Roma, adegua il suo progetto Il giardino romano tra il Cinque e Seicento al programma della Mostra con una sintesi ispirata all' "ordinata ricchezza dei motivi architettonici dei grandi giardini romani» di villa d'Este, villa Lante ed altre ville e giardini romani tre 'soo e '60o. All'architetto Tommaso Buzzi sono affidate due invenzioni, Il giardino veneziano del Settecento, "modellato sugli splendori delle ville Barbarigo a Valsanzibio e Pisani di Strà». Più sorprendente è la fonte che l'architetto dichiara aver utilizzato per Il giardino neoclassico lombardo ${ }^{20}$ per il quale si legge nella scheda di presentazione: "Qui s'è creato di fantasia, adoperando i preziosi elementi architettonici del centro da tavola di stile Impero ora nel Palazzo Reale di Milano ${ }^{21}$. L'idea rispetta alla lettera una delle istruzioni date ai comitati regionali secondo cui «anche alcuni centri tavola rappresentano giardini in piccolo». Nella presentazione delle Ville sabaude nel salone dei Dugento, Chevalley aveva lamentato la scomparsa di molti dei magnifici giardini di quelle Ville. Il suo Giardino piemontese del Settecento vuole:

[...] rappresentare, su un tipo ideale, fedeltà al gusto giardiniero del Piemonte, assai vicino al gusto dei giardini francesi. Le siepi di carpini tagliate a foggia di muro, con nicchie e passaggi come a Stupinigi, serrano il motivo centrale degli scomparti erbosi, dei rabeschi fioriti, degli agrumi in vasi di bronzo. In fondo al terreno, saliente sistemato a terrazze, la villa ricorda quella della Regina a Torino ${ }^{22}$.

19. Il giardino di Cosimo fu distrutto nel I5I8. Il progetto di Lusini è scelto tra i dieci presentati alla Mostra del I93I per essere restaurato alla fine degli anni ottanta. Si veda C. Acidini (a cura di), Giardini Medicei: giardini di palazzo e di villa nella Firenze del Quattrocento, Milano, Motta, 1996, pp. I38-I45.

20. <www.alinariarchives.it>: Mostra del giardino italiano I93I BGA-F-024274-0000.

2I. Ivi, p. 3I.

22. Ivi, p. 30 . 
L'ultima «invenzione» del Salone è infine il Giardino romantico ${ }^{23}$, cioè all'inglese, realizzato da Donatello Bianchini. Il modello deve confermare la fine della concezione classica e architettonica del giardino italiano sostituita da quella pittorica inaugurata nei giardini all'inglese e diffusasi successivamente in Francia e poi in Italia. Appare tuttavia, in primo piano, un «classico tempietto rotondo» che Ojetti aveva interpretato come «un rimpianto per l'architettura perduta», mentre il diorama doveva provare che «se la fase francesizzante può esser considerata ancora come uno sviluppo del vecchio tipo italiano, la fase inglese ne segna risolutamente il termine» ${ }^{24}$.

Nei progetti realizzati per la mostra i giovani architetti non osarono nessun tentativo di ricerca innovante sul tema dei giardino anche se non ignoravano il ricco scambio di esperienze artistiche apparso in Francia nel primo quarto del secolo. I futuristi Balla, Prampolini e Depero avevano presentato mobili ed oggetti alla mostra di Arti Decorative del 1925 a Parigi dove Mallet-Stevens e soprattutto Guevrekian avevano dato espressione ad una nuova progettazione del giardino suscitando polemiche. Era presente all'Expo. di Parigi anche Giò Ponti, medaglia d'oro all'Expo. per le sue ceramiche classicheggianti alla Richard Ginori. Date le loro posizioni d'avanguardia, di rottura degli schemi tradizionali e la loro negazione della storia i futuristi saranno esclusi dalla Mostra del I93I. Non si vedranno nella sezione dei «fiori finti ed anche contemporanei» nelle Sale dei Priori, le invenzioni colorate dei «fiori futuristi» creati prima degli anni Venti da Giacomo Balla, nessuno accenno al «fiore magico» del manifesto di Balla e Depero. Giò Ponti è ammesso nel comitato regionale della Lombardia perchè in quegli anni la sua posizione è vicina a quella del Gruppo degli architetti del Novecento e la sua produzione di ceramiche presso la Ginori è considerata rappresentativa di un'arte di lusso di cui Ojetti pubblica articoli elogiativi sulle pagine di «Dedalo». Il regime sembra non dare importanza al tema del giardino pubblico e del parco urbano che non considera come possibili mezzi di propaganda politica né come temi pubblicitari da sfruttare.

Nel periodo tra le due guerre la mostra non fu un modello per analoghe manifestazioni successive che saranno piuttosto dedicate alla fioricultura che al giardino. La maggior parte degli articoli apparsi su periodici e quotidiani nel 193I furono elogiativi. Tuttavia, la rivista «Architettura ed Arti decorative», diretta da Marcello Piacentini, dopo aver annunciato la

23. <www.alinariarchives.it>: Mostra del giardino italiano I93I BGA-F-024275-0000.

24. Ivi, p. 3 I. 
mostra nel suo numero di gennaio-febbraio 1931 e promesso di dedicare in seguito un intero numero all'avvenimento, non mantenne la promessa. Più polemica, "Casabella» manifestò critiche e dubbi. Mario Tinti pose al suo pubblico la domanda cruciale: "cosa significa tipicamente italiano?». Pur riconoscendo l'evidente sforzo d'erudizione esibito in tutte le sezioni della Mostra, Tinti resta scettico sulla capacità dell'evento a suscitare un risveglio nell'arte del giardino ${ }^{25}$. Può stupire anche il fatto che dei dieci grandi diorami creati come sintesi di ogni tipo di giardino italiano nelle varie epoche e nelle varie regioni d'Italia non ci sia stata alcuna immagine nel catalogo, né nelle riviste dell'epoca.

Malgrado le elaborate manipolazioni in stile degli architetti incaricati dei dieci diorami e la riunione dell'importante retroterra culturale formato dai documenti, molti dei quali di non facile accesso, perchè appartenenti a privati, la mostra non influenzò la ricognizione critica sul tema del giardino del periodo tra le due guerre. Il movimento di studi e revisioni sulla produzione artistica degli anni Trenta inizia verso la metà degli anni '70 quando appaiono i primi risultati di ricerca sotto forma di libri e convegni. Per l'arte dei giardini questo interesse appare più tardi. La mostra del I93I sarà riconsiderata come avvenimento culturale solamente a partire dalla metà degli anni ottanta quando si sviluppano anche le ricerche sul tema del giardino.

Il grande successo di pubblico della Mostra del i93i fu dovuto allo sforzo considerevole compiuto dagli organizzatori per ricomporre il particolare panorama iconografico disponibile sul tema. Sforzo a cui parteciparono, come ricorda Tarchiani, «un centinaio di commissari, sapienti, attivi e, quel che più conta, entusiasti della nostra idea» che "frugarono per mesi in tutta l'Italia». Questa dichiarazione di partecipazione entusiasta è senza dubbio da mettere sul conto dei risultati positivi della mostra. La riunione di numerosi documenti sui modi di rappresentare i giardini poteva soddisfare l'orgoglio patrimoniale del regime, il loro valore storico era invece dubbioso se utilizzati per stabilire i fondamenti storici dell'arte del giardini, i cui materiali sono essenzialmente mutevoli e deteriorabili. Contrariamente a quello che era successo per le mostre precedenti nel I9II e I922, nessuna pubblicazione sul tema de Il Giardino italiano farà seguito

25. «L'arte non si rinnovò mai, in nessun campo e in nessuna epoca, mercè siffatte abbondevoli imbandigioni di documenti e d'iconografie; e furono sempre di ben diversa natura le cause che determinarono nell'evoluzione dell'arte la sequela della tradizione e il fenomeno rinascimentale. Dal fiore risecchito, trattato alla formalina ed etichettato dall'erborista, non si videro mai nascere nuove specie di piante e di fiori: anche senza essere specialmente versati nella storia botanica e in quella del Giardino, questo lo si può affermare con certezza» (M. Tinti, «La Casabella», maggio I93I, n 4I, p. 39). 
alla Mostra del 193I, anche se Ojetti aveva annunciato il proposito di trarre da questa Mostra «un libro perchè dalla nostra fatica resti un durevole e utile ricordo». Nessuno tra i numerosi collaboratori si sentì in grado di fornire una sintesi storico-critica sul tema.

Ojetti aveva forse immaginato di aver raggiunto con successo gli obbietivi politici della Mostra volendo mostrare di aver «rimesso in onore un'arte singolarmente nostra». Cosa nella quale certamente riuscì. La mostra fu nazionalista nell'ossessionante celebrazione del valore patrimoniale italiano. Ojetti intendeva anche provare che l'arte dei giardini durante due secoli era stata della più alta qualità e che aveva anticipato molte opere di artisti inglesi e francesi. Su questo punto egli è perentorio. L'esposizione dimostrava, affermò, che anche durante il Settecento, nella Francia che aveva ereditato dall'Inghilterra la voga del giardino finto selvatico, «classici tempietti rotondi od ottagoni sorgevano ancora nel folto dell'elegante foresta romantica come un rimpianto per l'architettura perduta ${ }^{26}$. Una retorica che mirava ad arrestare ogni tentativo di abbandono delle classiche progettazioni ufficiali senza incoraggiare nuove ricerche sui temi del giardino e dei parchi pubblici di cui il Regime non potrà vantare nessuna importante realizzazione perché era stato il primo a disinteressarsene. 